\title{
Wind lens technology and its application to wind and water turbine and beyond
}

\author{
Yuji Ohya*, Takashi Karasudani, Tomoyuki Nagai, and Koichi Watanabe \\ Research Institute for Applied Mechanics, Kyushu University, 6-1 Kasuga-shi, Kasuga-koen, Fukuoka 816-8580, Japan
}

\begin{abstract}
Wind lens is a new type of wind power system consisting of a simple brimmed ring structure that surrounds the rotor causing greater wind to pass through the turbine. As a consequence, the turbine's efficiency of capturing energy from the wind gets dramatically increased. A Wind lens turbine can generate $2-5$ times the power of an existing wind turbine given at the same rotor diameter and incoming wind speed. This fluid dynamical effect is also effective in the water. We have developed 1-3 kW Wind lens turbines and a $100 \mathrm{~kW}$ Wind lens turbine. In addition to the enhanced output power, Wind lens turbine is quiet. The technology is now used in an offshore experiment with a hexagonal float 18 meters in diameter set off the coast of Hakata Bay in Fukuoka City. Moreover, we are now pursuing larger size Wind lens turbines through multi-rotor design consisting of multiple Wind lens turbines in a same vertical plane to embody larger total power output.
\end{abstract}

\section{Introduction}

We have been investigating the efficient utilization of fluid energy at the Research Institute for Applied Mechanics (RIAM), Kyushu University. In this report, our new technology of enhancing fluid power generation system and related recent activities of our lab will be introduced. We have developed a new wind turbine system that consists of a diffuser shroud with a broad-ring brim at the exit periphery and a wind turbine inside it. The shrouded wind turbine with a brimmed diffuser has demonstrated power augmentation by a factor of about 2-5 compared with a bare wind turbine, for a given turbine diameter and wind speed [1]. We named the new turbine "Wind lens turbine". At this moment (July 2015), small Wind lens turbines of $1-3 \mathrm{~kW}$ and a mid-size of $100 \mathrm{~kW}$ Wind lens turbine have been developed for practical application. Aiming at harvesting offshore wind energy, this technology is applied in our floating platform renewable energy farm experiment equipped with the Wind lens turbines $(3 \mathrm{~kW} \times 2$ units) and PV panels $(2 \mathrm{~kW})$ in Hakata Bay, Fukuoka Japan. In recent years, our concept of the research on renewable energy is based on a key word of "Small is profitable". Considering the social acceptability and environmental problems, we have been developing a multi rotor system (MRS), namely a clustered Wind lens turbine in one vertical plane to obtain larger power output.

The mechanism of the Wind lens can be applied in the water also. Development of shrouded water turbine is ongoing at Kyushu University. A water channel experiment

\footnotetext{
* e-mail: ohya@riam.kyushu-u.ac.jp
}

with small Water lens turbine demonstrated 2.5-time power enhancement using the same diffuser design used in the Wind lens turbine [2].

\section{What is the Wind lens turbine?}

\subsection{The idea of a shrouded diffuser with brim}

Wind power is proportional to the wind speed cubed. If we can increase the wind speed with some mechanism by utilizing the fluid dynamic nature around a structure, namely if we can capture and concentrate the wind energy locally, the output power of a wind turbine can be increased substantially [1]. There appears hope for utilizing the wind power in a more efficient way. For this purpose, we have developed a diffuser-type structure that is capable of collecting and accelerating the approaching wind. We have devised a diffuser shroud with a large brim that is able to increase the wind speed from approaching wind substantially by utilizing various flow characteristics, e.g., the generation of low pressure region by vortex formation, flow entrainment by vortices and so on, of the inner or peripheral flows of a diffuser shroud, as shown in Figure 1. Although the concept of ducted or shrouded wind turbine has existed since mid20th century, the key element that distinguishes Wind lens from the others is a large brim attached at the exit of diffuser shroud. A number of field test was carried out with the Wind lens turbines, and it demonstrated power augmentation for a given turbine diameter and wind speed by a factor of about 2-5 compared to a standard wind turbine. Figure 1 shows the power enhancement by one of the most compact Wind lens model. Larger Wind lens produces higher efficiency. However, larger diffuser has its cons such as increased wind load and structural weight in terms of practical application. 


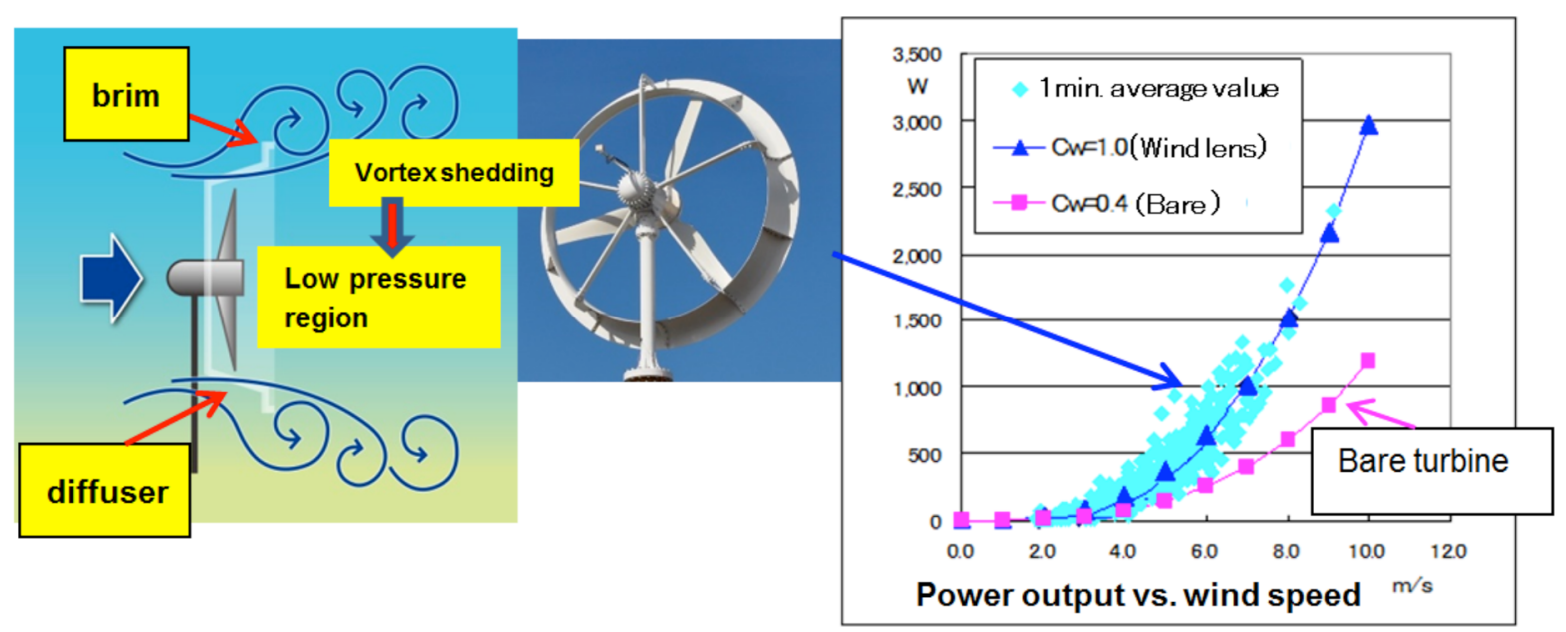

Fig. 1. Wind lens turbine, the mechanism and its performance.

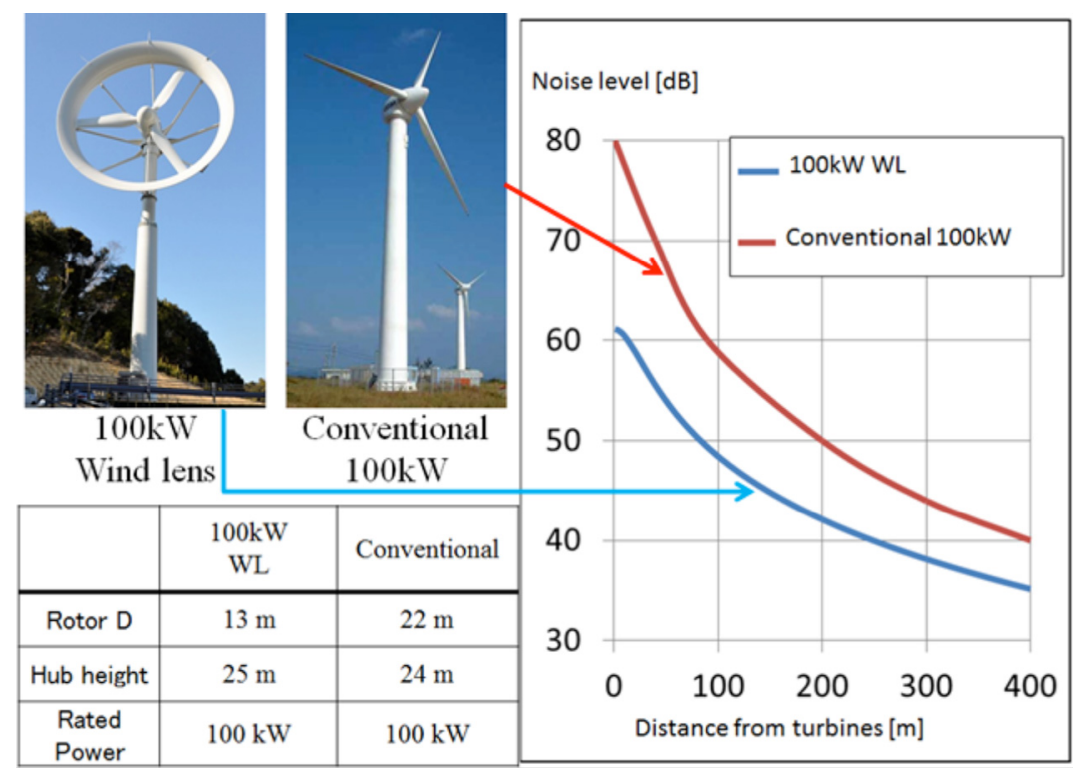

Fig. 2. Noise comparison between a Wind lens and a conventional turbine $(100 \mathrm{~kW})$.

\subsection{Characteristics of a wind turbine with brimmed diffuser shroud}

The list of important features of the Wind lens turbine includes,

- Two-fivefold increase in output power compared to conventional wind turbines with the same rotor diameter due to concentration of the wind energy.

- Brim-based yaw control: The brim at the exit of the diffuser makes the turbine rotate following the change in the wind direction, like a weathercock. As a result, the wind turbine automatically turns to face the wind.

- Significant reduction in wind turbine noise (Fig. 2): Basically, an airfoil section of the turbine blade, which gives the best performance in a low-tip speed ratio range, is chosen. Since the vortices generated from the blade tips are considerably suppressed through the interference with the boundary layer within the diffuser shroud, the aerodynamic noise is reduced substantially $[3,4]$.
- Improved safety: The wind turbine, rotating at a high speed, is shrouded by a structure and is also safe against damage from broken blades.

- As for demerits, wind load to a wind turbine and structural weight are increased compared to the conventional turbines with the same rotor diameter.

This Wind lens technology is also applicable to water turbines. We have recently showed the similar improvement in efficiency using a prototype of Water lens turbine using a water channel [2]. This experiment is the first step toward the development of micro hydro power generation system and tidal current power generation system that are now under development at Kyushu University.

\subsection{Mid-size next generation Wind lens project in Kyushu University}

For the purpose of practical application to a small- to midsize wind turbine, we have developed a very compact Wind lens diffuser to reduce the wind load and the structural 
Total Power Output of 3 WLT in multi rotor arrangement

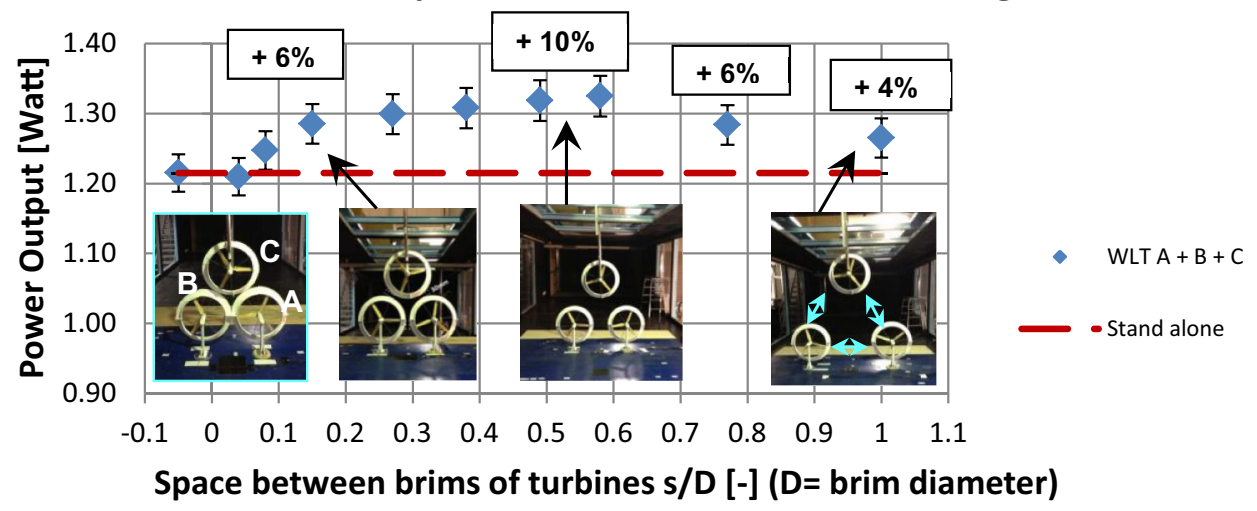

Fig. 3. Total power output of 3 Wind lens turbines in multi rotor arrangement. The red broken line indicates the sum of each power output of stand-alone turbines.

weight that lead to the reduced production cost. Using the compact diffuser, we have achieved a two- or three-fold increase in output power as compared to conventional (bare) wind turbines with the same rotor diameter, as shown in Figure 1. We also developed a prototype Wind lens turbine of $100 \mathrm{~kW}$ at a rated wind speed of $12 \mathrm{~m} / \mathrm{s}$ with this compact diffuser. The rotor diameter is $12.8 \mathrm{~m}$, which is much smaller than that of a conventional wind turbine with the same rated power, say, two-thirds in the rotor size, as shown in Figure 2. This turbine employs a fixed blade pitch system and a passive (free) yawing system as used in smaller Wind lens turbine models. Currently, more advanced $100 \mathrm{~kW}$ Wind lens turbine is being designed and under development. This new model will have active pitch control system and semi-active yawing system. This new $100 \mathrm{~kW}$ Wind lens turbine will be also a part of multi rotor system (MRS) for larger rated power output.

\section{Clustering of Wind lens turbines to a multi rotor system (MRS)}

The wind turbine industry has seen innovations leading to growing size of turbines of currently over $140 \mathrm{~m}$ in diameter for multi-MW wind turbines. This global trend is driven to reduce the energy production cost per unit electric power, and has been supported by the advancing technology of lighter and stronger materials, e.g., Carbon Fiber Reinforced Plastic (CFRP). However, as pointed out in some recent studies, scaling of blades has its limitations and therefore advantages of multi-rotor system concepts have been suggested by Jamison et al. [5]. Multi rotor turbines are turbine systems comprising multiple rotors in one structure. Theoretically, a MRS turbine consisting of $n$ unit turbines weighs $1 / \sqrt{n}$ of a single turbine of the same rated power. We have been investigating the aerodynamics of Wind lens turbines spaced closely together in a variety of multi rotor arrangements. The focus began on three turbines in a triangle arrangement and now the investigation is expanded as large as seven-rotor design. In the future the number will increase even more. In three-turbine case, a number of different Wind lens turbine configurations have been investigated, mainly varying the brim height between

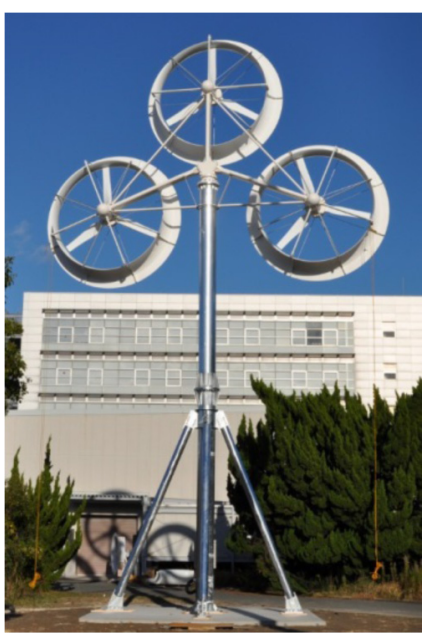

Fig. 4. $10 \mathrm{~kW}$ multi rotor Wind lens turbine system $(3 \mathrm{~kW} \times 3)$ at Kyushu University.

$3 \%$ and $20 \%$ of the rotor diameter and the geometry of the triangle, e.g., opening angle and spacing, etc. In wind tunnel experiments we discovered that closely spaced turbines have an influence on each other's power output. As the separation gaps are increased, the total system power output can exceeded the sum of each power output of the stand-alone turbines as shown in Figure 3. Further, it was observed that the individual power of a turbine output does not follow the trend of the cumulative power output. These phenomena can be explained with flow patterns observed in gap flow analysis of bluff bodies, as discussed in the reference [6]. A field test has started with small full scale Wind lens turbines at RIAM campus of Kyushu University. Figure 4 shows the $10 \mathrm{~kW}$ MRS Wind lens turbine with three $3 \mathrm{~kW}$ turbines.

\section{Offshore floating energy farm}

\subsection{Floating energy farm: a pilot plant in Hakata Bay, Fukuoka}

A small offshore hybrid energy farm experiment in Hakata Bay, Fukuoka, Japan started as our first step toward realizing next generation mid-size distributed offshore 


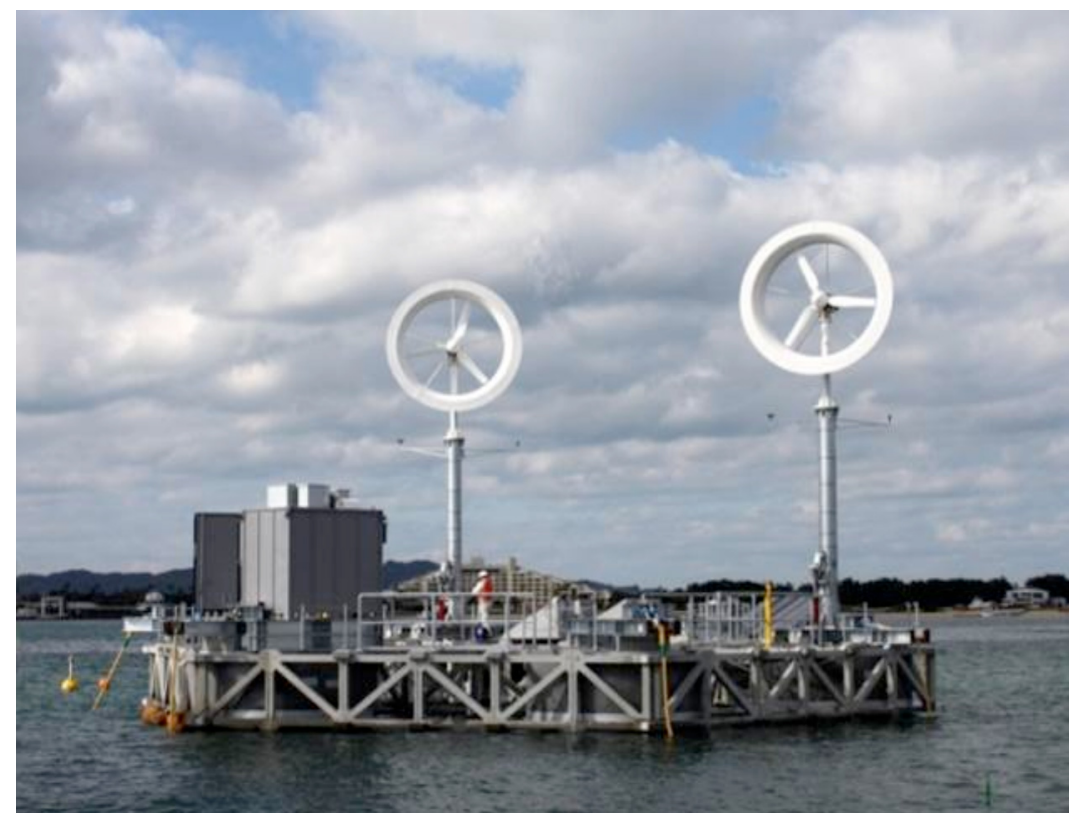

Fig. 5. Eighteen meter wide concrete platform of offshore hybrid farm experiment by Kyushu University at Hakata Bay, Japan.

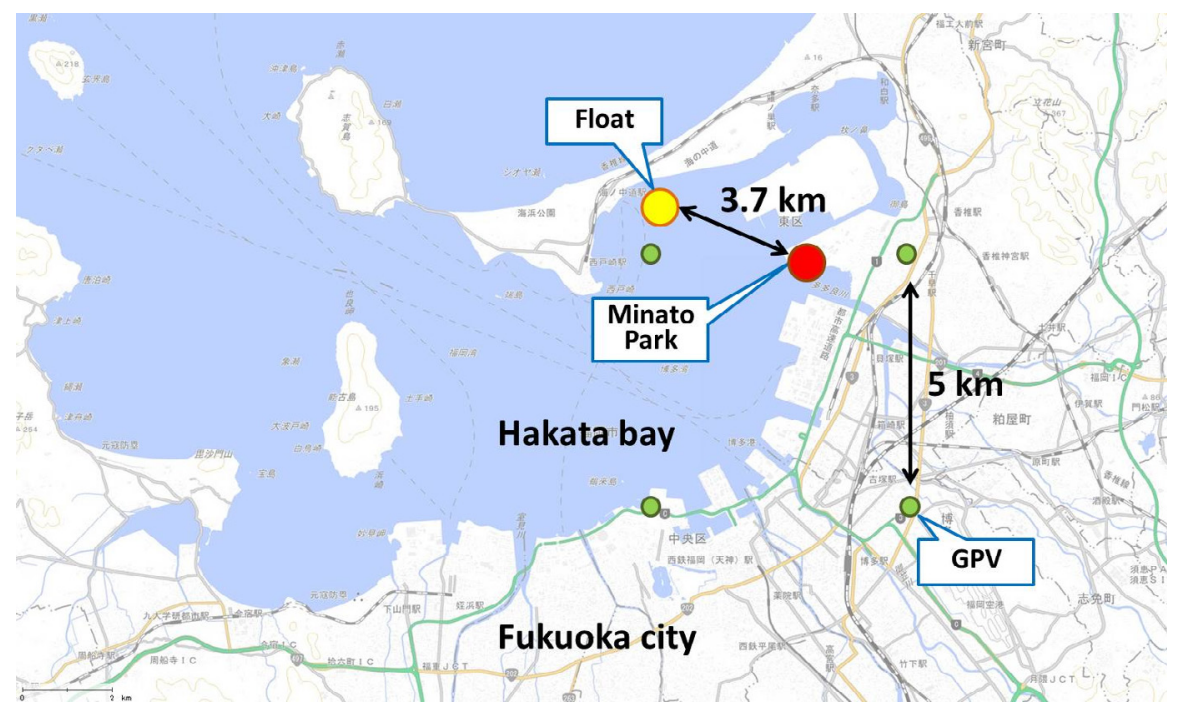

Fig. 6. Mooring location of the platform for the Hakata-Bay experiment. There is a bay side park called "Minato Park" on the adjacent shore, $3.7 \mathrm{~km}$ away from the platform.

renewable energy farm. One of the main goals of this experiment was to clarify the advantage of wind farming at offshore locations, even relatively short distances from the adjacent shore, with typical annual average wind speed in Japan. A floating platform was constructed and equipped with two of $3 \mathrm{~kW}$ Wind lens turbines together with $2 \mathrm{~kW} \mathrm{PV}$ panels. In early December 2011, the $18 \mathrm{~m}$ wide hexagonal floating pre-stressed concrete platform was launched and moored 800 m off the coast of Fukuoka, as shown in Figures 5 and 6 . The wind speed and corresponding power production were compared to a similar land based Wind lens turbine system on the adjacent seaside park called "Minato Park", that is $3.7 \mathrm{~km}$ away from the platform.
Data from a year of operation (from November 2012 to October 2013) showed higher average wind speed through the period resulting doubled power production for the offshore turbines (Figs. 7 and 8). This comparison shows a clear advantage for offshore wind farming. This result might appear very natural and insignificant since it is widely known that the offshore areas usually provide better wind conditions for turbines due to smaller surface roughness. However, there are very limited numbers of studies in which the wind conditions and corresponding wind turbine power output from identical system installed at both offshore point and land based point are compared over the same extended period of time. 


\subsection{The second stage of the floating energy farm plan}

We are planning to expand this experiment to a larger size for more practical application. As shown in Figure 9, a floating body of $\sim 70 \mathrm{~m}$ wide triangular semi-submersible structure will be the unit structure of the second stage

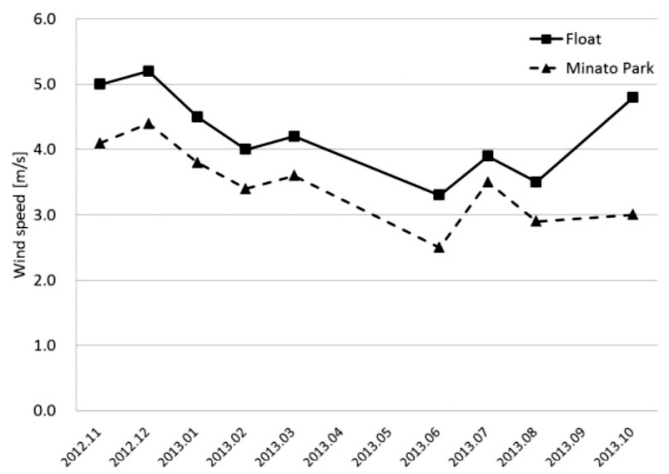

Fig. 7. Monthly average wind speed comparison between the offshore platform and Minato Park.

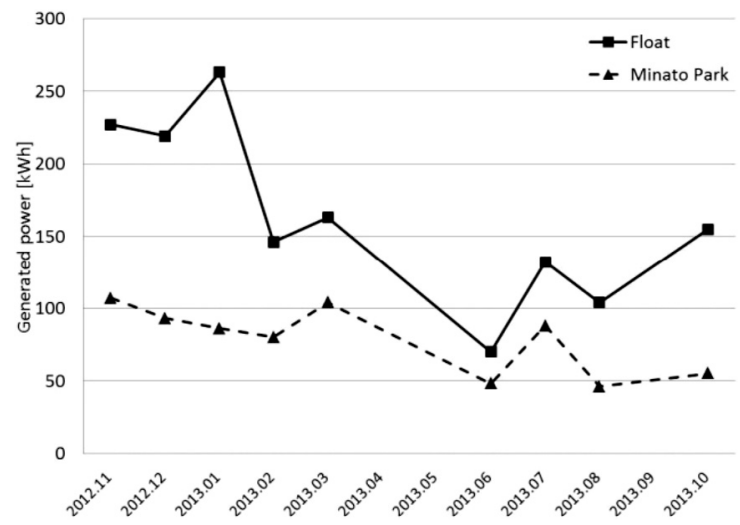

Fig. 8. Monthly power output comparison between the offshore platform and Minato Park. offshore farm plan following the successful result of the Hakata-Bay pilot plant. It can be expanded into multitriangle body. This farm will accommodate medium size (300 kW at a wind speed of $12 \mathrm{~m} / \mathrm{s}$ ) Wind lens turbines according to the current plans. This platform is designed to suit the installation within a few kilometers from adjacent shore of small islands or small isolated villages in Japan. A semi-automated marine farm is also planned to be installed in and around it. Therefore the quietness of the Wind-lens turbines will become a key aspect of the success of the plan. We are aiming at the realization of a fishery-harmonious floating renewable energy platform.

\section{Conclusion}

The Wind lens technology developed at the Kyushu University demonstrated high power efficiency by active utilization of vortex shedding behind the brim. This unintuitive mechanism induces low pressure region behind the structure causing more wind flow into the rotor. The list of advantages also includes improved quietness due to cancellation of the blade tip vortex. This quietness enables the installations of the turbine relatively close to the residential areas. Therefore, even for larger size turbines, it seems possible to realize near shore mid-size hybrid energy farm. In Japan most of the coastal areas are populated. Therefore conventional large scale offshore farm must be placed far from the shore (usually as far as $10 \mathrm{~km}$ from the shore) to avoid low frequency noise issues that often become controversial for the nearby residents.

We are also developing larger Wind lens turbine system through multi rotor design. This system has many advantages such as reduced total mass leading to cost reduction compared to single turbine design with the same rated power output. Moreover, Wind lens multi rotor system can enhance total power output as a whole due to interactions of flows between neighboring Wind lens turbines. System optimization with three turbines has started in our wind tunnel, and it has shown

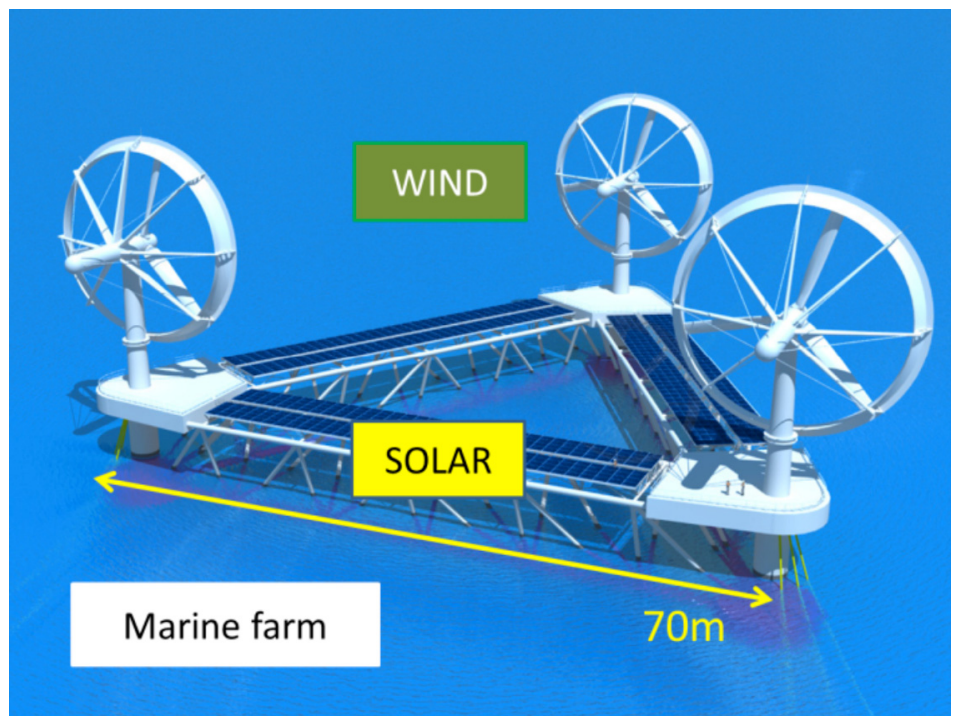

Fig. 9. Concept image of the next generation mid-size offshore hybrid energy farm (second stage). A side spans about $70 \mathrm{~m}$ to form a triangular platform hosting three $300 \mathrm{~kW}$ Wind lens turbines and PV panels making total rated output as large as 1 MW scale. 
encouraging experimental results. This experiment is still on going with more complicated configuration with increased number of turbines.

This study was partially supported by Ministry of the Environment Japan. We gratefully acknowledge Kyushu University, and our laboratory staff Kimihiko Watanabe. We also would like to acknowledge kind cooperation by city of Fukuoka.

\section{References}

1. Y. Ohya, T. Karasudani, A shrouded wind turbine generating high output power with Wind lens technology, Energies 3, 634 (2010)
2. H. Sun, Y. Kyozuka, Analysis of performances of a shrouded horizontal axis tidal turbine, in Conference paper of OCEANS, May 2012 (2012), doi:10.1109/OCEANSYeosu.2012.6263455

3. K. Abe et al., An experimental study of tip-vortex structures behind a small wind turbine with a flanged diffuser, Wind Struct. 9, 413 (2006)

4. S. Takahashi et al., Behaviour of the blade tip vortices of a wind turbine equipped with a brimmed-diffuser shroud, Energies 5, 5229 (2012)

5. P. Jamieson, M. Branney, Multi-rotors; a solution to $20 \mathrm{MW}$ and beyond? Energy Procedia 24, 52 (2012)

6 . Y. Ohya, Bluff body flow and vortex - its application to wind turbines, Fluid Dyn. Res. 46, 061423-1 (2014)

Cite this article as: Yuji Ohya, Takashi Karasudani, Tomoyuki Nagai, Koichi Watanabe, Wind lens technology and its application to wind and water turbine and beyond, Renew. Energy Environ. Sustain. 2, 2 (2017) 